\title{
Design and Development of Intelligent Vending Cabinet System
}

\author{
Mengfei Gao \\ Electrical Engineering College, Southwest Minzu University, Chengdu, China
}

\begin{abstract}
Common vending cabinets prevalent in today's market precludes distant knowledge about the actual sales of a specific cabinet, thereby necessitating the daily inspection of all the cabinets by the administrator. The designing of a new vending cabinet that allows the administrator to replenish goods based on the system data will save labor costs and improve profits.
\end{abstract}

Keywords: Intelligent Vending Cabinets, Applet, System Design.

\section{Introduction}

With the development of IoT (Internet of Things) and AI technologies, self-service intelligent retail has gradually become a part of our life. Many medium and large cities in China have emerged to be new markets for unmanned retail as self-service, intelligence and automation continuously shape people's life style.

In 2017, unmanned shelves set off an orgy [1] that attracted countless sellers and investors to join. However, as the enthusiasm hastily grew, its collapse soon arrived. Problems such as high commodity damage rate, failure in delivery, and unsatisfactory profitability gradually surfaced, and the unmanned shelf market quickly cooled.

As the Covid-19 pandemic gradually becomes a normality, if we can develop a new type of intelligent vending cabinets that solve the difficulty in the last $100 \mathrm{~m}$ delivery caused by insufficient deployment of conventional cabinets, we will enable minimum or none human touch in a true sense, thus making contributions to the control of the pandemic. Meanwhile, an innovative consumption mode will be brought about to truly realize consumption upgrade.

\section{System Design and Implementation Analysis}

\subsection{Design of System Main Body}

(1) Considering that the cabinets are distributed in different cities and scenarios, in order to prevent the possible troubles caused by overlapping management and maloperation by the staff, it is necessary to implement administration over all the users to realize division of the users and to enforce different user permissions [2].

a. Personnel management: Information such as region, identity information, ID number of the members needs to be included to set different user permissions. Users shall use the system with the scope of permission.

b. Permission management: Complete the assignment of different levels to different users, and give permission according to the levels in order to prevent loopholes in the system.

(2) Management of the hardware cabinets is a basic function, so it is a must to realize cabinet management, such as information viewing, modification of cabinet ID, status query, and cabinet adding. Hardware cabinet management is specially provided to O\&M staff for them to realize the replenishment and cleaning of goods and the maintenance of hardware.

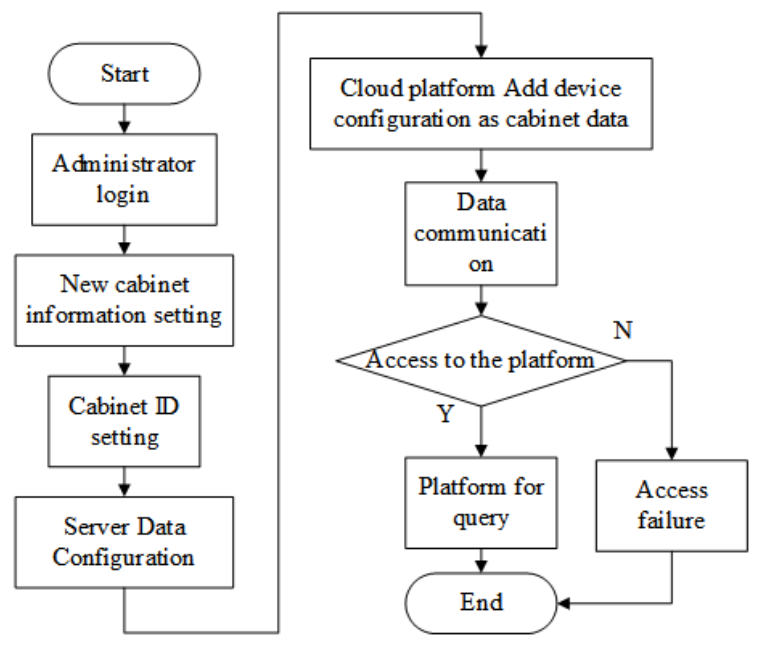

Figure 1. Management of Hardware Cabinets

(3) User control management is the key function of the system, which allows a series of operations through WeChat, including controlling the opening/closing of the cabinet door, paying bills and other functions.

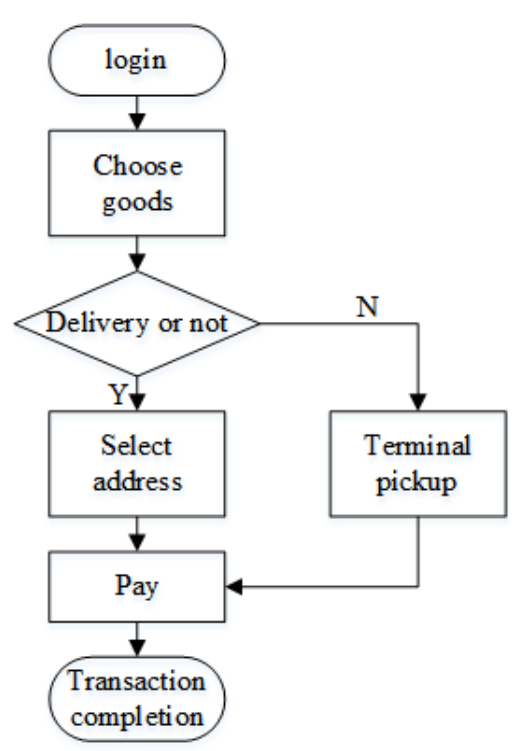

Figure 2. User Control Management 
(4) Construct an IoT platform [3] to enable communication between IoT and the server, forwarding of data instructions and storage of data in the server. Design the IoT application server and provide web pages and WeChat services. Conclude the development of a complete cloud platform of IoT.

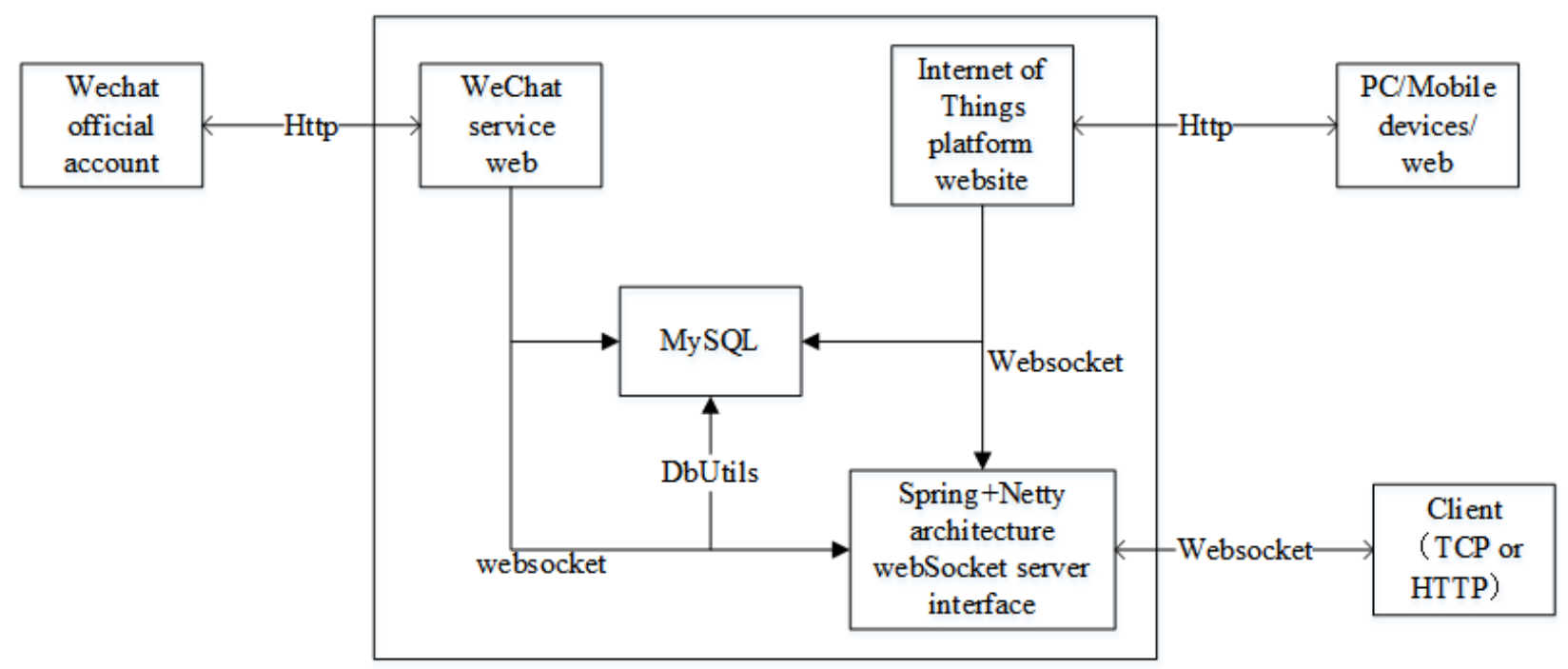

Figure 3. IoT Service Platform

\subsection{Analysis of System Realization}

(1) Analysis of the realization of administrator privileges. Every administrator has their own user ID and password, and personnel permissions have been assigned at the very beginning. As shown in Figure 6, administrators have privileges unique to them.

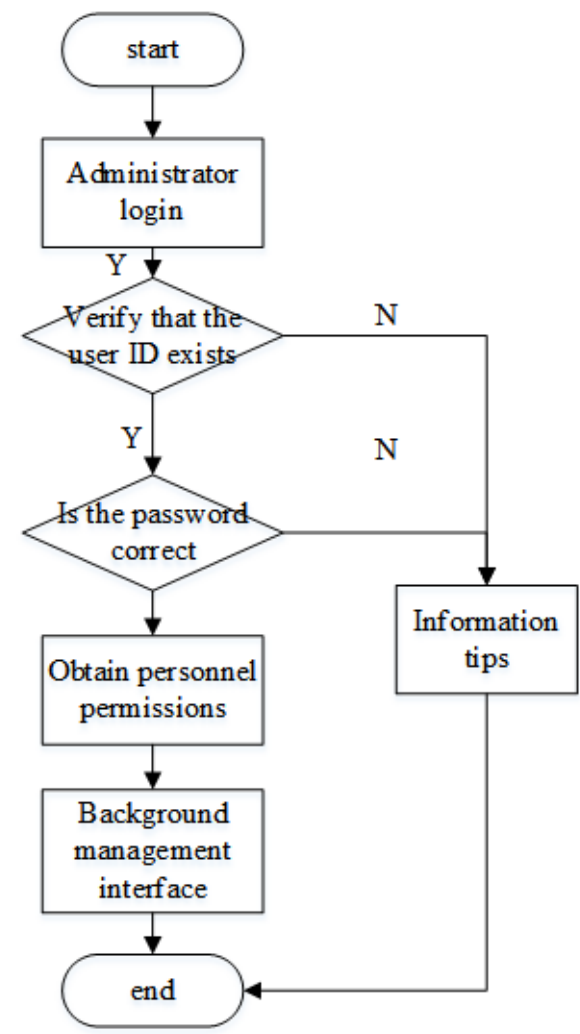

Figure 4. Analysis of the Realization of Administrator Permission

(2) Analysis of the process of user operation. The user sends the request of operating the cabinet through WeChat. After the cloud platform receives the request, it interprets the request and relevant data, confirms the operation, and sends the feedback result to the user's WeChat. The user confirms through WeChat and sends the request again. After receiving the request, the cloud platform operates through the communication interface program and sends instructions to the network module. The cabinet terminal performs the operation, and the result is then fed back to the communication module. The communication module further feeds back the result to the cloud platform, and finally the result is fed back to the user's WeChat so that the entire operation process is completed.

\section{Experimental Results of the System}

With the help of the WeChat applet, users can directly scan the QR code on the cabinet to make purchases. They can also purchase the products they want by tapping the "search" button.

After they scan the QR code, they can gain access to the product page linked to different machines and make purchases. All they have to do is select the product they need, submit the order and open the cabinet.

There are two ways for users to log in. They can either type into their phone number and verification code, or tap the WeChat authorization button.

As soon as they log in, they can search for the product, and vending machines at different addresses will be recommended based on the searched product. Users can then select the product. After they make the selection, they will arrive at the page listing the details of the order where they can see the product they have selected, the fee and address of the delivery etc., and they can make online payment here.

Users can add new addresses, and accurate positioning with the help of online map is also available here.

After the order is completed, users can leave their comments on the delivery and the seller's services through the commenting system, so that the admistrator can receive the feedback in time. 


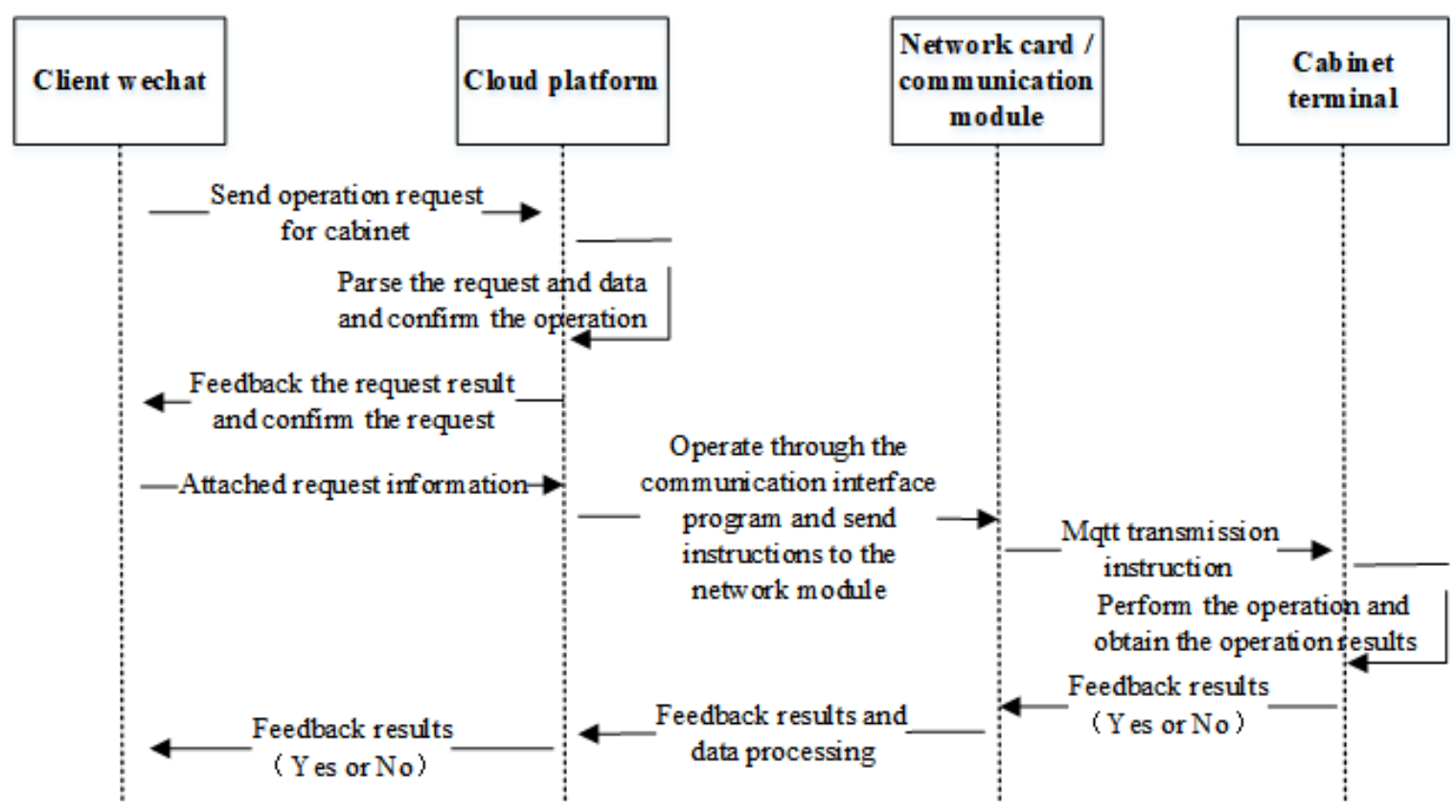

Figure 5. Analysis of the Process of User Operation

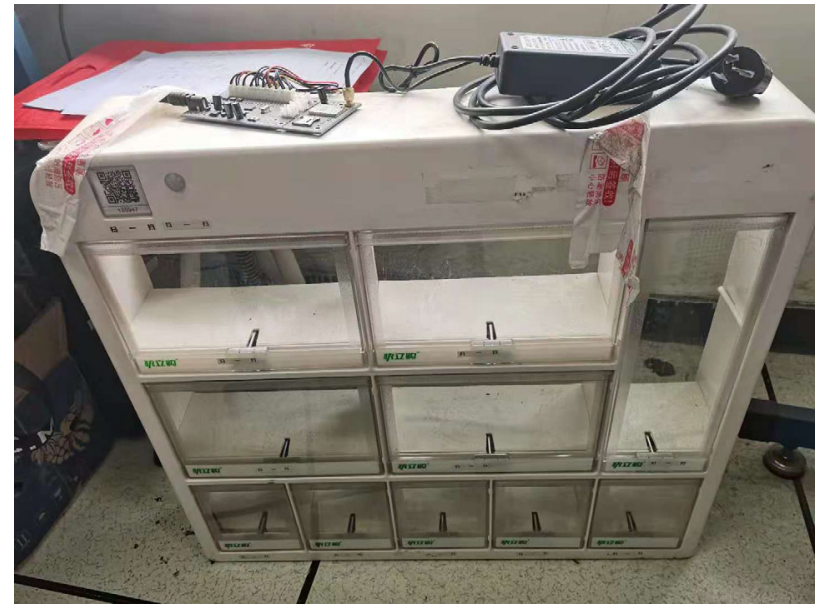

Figure 6. Real Example of the Cabinet

\section{Conclusion}

By developing a new type of intelligent vending cabinets, we try to solve the difficulty in the last $100 \mathrm{~m}$ delivery caused by insufficient deployment of conventional cabinets, and enable minimum or none human touch in a true sense, thus making contributions to the control of the pandemic. Meanwhile, an innovative consumption mode will be brought about to truly realize consumption upgrade.

\section{Acknowledgment}

This project is supported by the Graduate Innovative Scientific Research Project of Southwest Minzu University (Design and Development of Intelligent Vending Cabinet System, Item no:CX2021SP104).

\section{References}

[1] Winnders Hard to Find in Smart Cabinets [J]. Invention and Innovation, Big Tech. 2019(7): 38-39.

[2] Tan Yi. Design of Shared Cabinet System Based on Internet of Things [D]. Guangdong University of Technology, 2019. DOI: 10.27029/d.cnki.ggdgu.2019.000936.

[3] Liang Yingnan. Research of Commodity Recognition Algorithm in the Scenario ofIntelligent Cabinet and System Design [D]. South China University of Technology, 2020. DOI: 10.27151/d.cnki.ghnlu.2020.005109. 